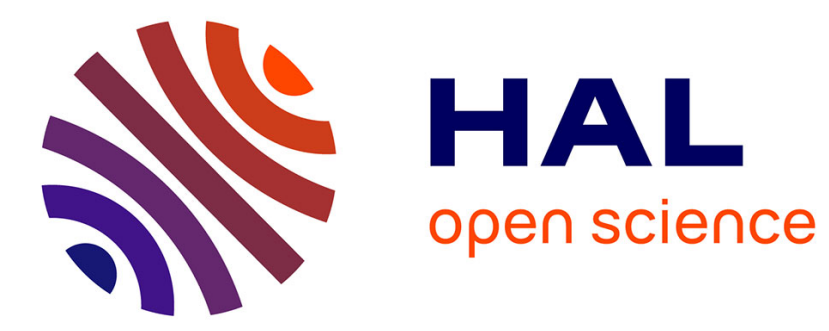

\title{
Spatio-temporal registration of 3D microscopy image sequences of Arabidopsis floral meristems
}

Gaël Michelin, Yassin Refahi, Raymond Wightman, Henrik Jönsson, Jan J. Traas, Christophe Godin, Grégoire Malandain

\section{- To cite this version:}

Gaël Michelin, Yassin Refahi, Raymond Wightman, Henrik Jönsson, Jan J. Traas, et al.. Spatiotemporal registration of 3D microscopy image sequences of Arabidopsis floral meristems. ISBI - International Symposium on Biomedical Imaging, Apr 2016, Prague, Czech Republic. pp.4, 10.1109/ISBI.2015.7163872 . hal-01606687v2

\section{HAL Id: hal-01606687 \\ https://inria.hal.science/hal-01606687v2}

Submitted on 22 Apr 2016

HAL is a multi-disciplinary open access archive for the deposit and dissemination of scientific research documents, whether they are published or not. The documents may come from teaching and research institutions in France or abroad, or from public or private research centers.
L'archive ouverte pluridisciplinaire HAL, est destinée au dépôt et à la diffusion de documents scientifiques de niveau recherche, publiés ou non, émanant des établissements d'enseignement et de recherche français ou étrangers, des laboratoires publics ou privés. 


\title{
SPATIO-TEMPORAL REGISTRATION OF 3D MICROSCOPY IMAGE SEQUENCES OF ARABIDOPSIS FLORAL MERISTEMS
}

\author{
Gaël Michelin ${ }^{\star 1} \quad$ Yassin Refahi ${ }^{\dagger} \quad$ Raymond Wightman ${ }^{\dagger}$ \\ Henrik Jönsson ${ }^{\dagger} \quad$ Jan Traas $^{\ddagger} \quad$ Christophe Godin ${ }^{\S 2} \quad$ Grégoire Malandain $^{\star 1}$ \\ ${ }^{\star}$ Inria, 06900 Sophia Antipolis, France \\ ${ }^{\dagger}$ Sainsbury Laboratory, University of Cambridge, Cambridge CB2 1LR, UK \\ ${ }^{\ddagger}$ Laboratoire RDP, Université de Lyon 1, ENS-Lyon, INRA, CNRS, 69364 Lyon, France \\ ${ }^{\S}$ Inria, 34095 Montpellier, France
}

\begin{abstract}
The shoot apical meristem (SAM) is at the origin of all the plant above-ground organs (including stems, leaves and flowers) and is a biological object of interest for the understanding of plant morphogenesis. The quantification of tissue growth at a cellular level requires the analysis of $3 \mathrm{D}$ microscopic image sequences of developing meristems. To address inter-individual variability, it is also required to compare individuals. This obviously implies the ability to process inter-individual registration, i.e. to compute spatial and temporal correspondences between sequences from different meristems. In the present work, we propose a spatial registration method dedicated to microscopy floral meristem (FM) images, and the identification, for a given still image of a meristem, of its best corresponding time-point in a sequence of an other individual (temporal registration).
\end{abstract}

Index Terms - Confocal microscopy, developmental biology, floral meristem, Arabidopsis thaliana

\section{INTRODUCTION}

In developmental biology, the study of growing organisms at cell level for the understanding of tissue morphogenesis is necessary for the study of underlying genetic mechanisms that govern the development. The imaging techniques rapidly progressed over the last decades, and it becomes now possible to study both animal and plant growth by imaging in vivo temporal $3 \mathrm{D}$ stacks of respectively embryos and meristems with excellent spatial resolution and with an imaging frequency that is good enough to follow the organism deformations over the time, at cell level [1]. One central aim in morphogenesis study is to compare individuals in order to understand the impact of genetic backgrounds on the organism growth. To do so, there is a need for inter-individual registration tools since it would open the way to do quantified statistical studies of development variability. There exists some attempts to develop such inter-individual registration on these new types of $3 \mathrm{D}+t$ developmental data on embryos $[2,3]$, which exhibit a stereotyped development in the early stages, but not on more complex organisms. In this article, our aim is to propose a method that opens the way to build such a tool applied to floral meristems (FM), which is one of the above-ground organs of the plant elaborated by the shoot apical meristem (SAM).

\footnotetext{
Contact: \{gael.michelin,gregoire.malandain\}@inria.fr

${ }^{1} \mathrm{GM}$ and GM are with Inria/I3S/iBV project-team Morpheme, Sophia Antipolis, France.

${ }^{2} \mathrm{CG}$ is with Inria project-team Virtual Plants, Montpellier, France.
}

We reduce here the problem of comparing two temporal sequences of 3D images of different individuals into a comparison between a still (reference) 3D image and a temporal (test) sequence of 3D images, since a sequence-to-sequence registration tool can be directly deduced from an image-to-sequence one. The meristem is compounded of cells grouped in distinct layers (L1, L2, L3) where L1 and L2 are respectively the epidermal and the upper hypodermal layer. The cells in L1 and L2 divide anticlinally, i.e. in a sideway fashion that ensures that L1 and L2 remain distinct [4]. Meristem development is not stereotyped at the cellular level, implying there is no cell-to-cell mapping between meristems at the same development stage. Nevertheless flowers from the same species display a great robustness in their global shape [5] and the meristem development stage is identifiable to its size [6]. Thus a goodness-of-fit criterion on L1 and L2 layer interfaces can be considered as an adequate registration quality measure.

Registering 3D images of FM comes then to register the $\mathrm{L} 1$ and L2 layers: surface extraction will be described in section 2 while surfaces spatial registration will be addressed in section 3. Given a still 3D image, identifying its corresponding time-point (temporal registration) in an other $3 \mathrm{D}+t$ sequence is achieved by selecting the "best" spatial registration of the still image with respect to all images of the sequence, as detailed in section 4. Experimental results processed on Arabidopsis thaliana microscopy image sequences are presented in section 5 .

\section{FEATURES EXTRACTION}

In this section, $I$ denotes a floral meristem image. Here, we consider that the cell segmentation of $I$ is well known (one can refer to [7, 8] for cell segmentation frameworks). The $\mathrm{L} 1$ cells are easily extracted as they are the only ones in contact with the image background, and similarly L2 cells are extracted as they constitute the L1 sub-layer. We define the following notations:

- $k=1$ (respectively 2,3 ) denotes the background-L1 (respectively the L1-L2, the L2-L3) interface;

- $K$ denotes a set of interfaces (e.g. $K=\{1,2\}$ );

- $S_{k}^{I}$ is the surface at the interface $k$;

- $S_{K}^{I}$ is the set of surfaces $\left\{S_{k}^{I}\right\}_{k \in K}$;

- $P_{k}^{I}$ is a point-set sampling $S_{k}^{I}$;

- $P_{K}^{I}$ is the set of point-sets $\left\{P_{k}^{I}\right\}_{k \in K}$.

Fig. 1 illustrates the $P_{K}^{I}$ extraction process for $K=\{1,2\}$. 


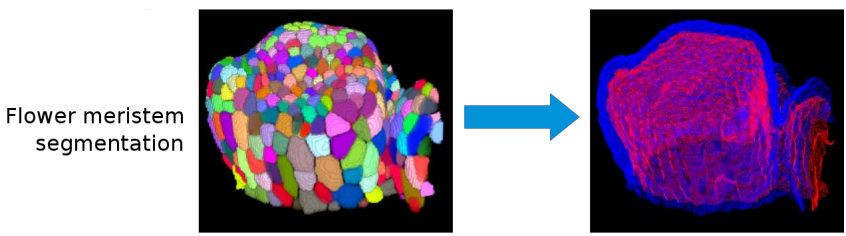

Fig. 1: Segmentation of a FM image $I$ (left) and the point-sets $P_{K}^{I}$ extraction result for $K=\{1,2\}$ (right). The background-L1 (resp. L1-L2) interface points are displayed in blue (resp. in red).

\section{SPATIAL REGISTRATION}

We consider here two point-sets $P_{K}^{I}$ and $P_{K}^{J}$. Spatial registration consists in computing the (here affine) optimal transformation $A_{I \leftarrow J}^{\mathrm{opt}}$ that registers $P_{K}^{J}$ on $P_{K}^{I}$ with respect to a point-sets distance $d$ :

$$
A_{I \leftarrow J}^{\mathrm{opt}}=\arg \min _{A \in \operatorname{Aff}\left(\mathbb{R}^{3}\right)} d\left(P_{K}^{I}, A\left(P_{K}^{J}\right)\right) .
$$

To do so, we apply a robust version of the Iterative Closest Points (ICP) algorithm [9] with a trimmed Least Squares Estimation (LSE) [10]. The following steps are thus processed:

1. Initialization of the transformation $A:=A_{I \leftarrow J}^{\text {init }}$.

2. Do until convergence of $A$ :

(a) Computation of the points pairing function $\phi_{A}: P_{K}^{J} \rightarrow P_{K}^{I}$ so that for $k \in K$ and $p \in P_{k}^{J}$, $\phi_{A}(p)=\arg \min _{q \in P_{k}^{I}} \operatorname{dist}(q, A(p))$,

i.e. $\phi_{A}(p)$ is the nearest neighbor of $A(p)$ in $P_{k}^{I}$ with respect to the Euclidean distance.

(b) Refining of the linear transformation

$$
A:=\arg \min _{A^{\prime} \in \operatorname{Aff}\left(\mathbb{R}^{3}\right)} r_{\text {trim }}\left(\left\{\phi_{A}(p), A^{\prime}(p)\right\}_{p \in P_{K}^{J}}\right) .
$$

3. Let us denote $A_{I \leftarrow J}^{\mathrm{opt}}=A$ at the end of the process.

In this process, $r_{\text {trim }}$ denotes the trimmed average of the square residuals of a set of paired points:

$$
r_{\text {trim }}\left(\left\{\phi_{A}(p), A^{\prime}(p)\right\}_{p}\right)=\frac{1}{\# \widetilde{P}_{K}^{J}} \sum_{p \in \widetilde{P}_{K}^{J}} \operatorname{dist}\left(\phi_{A}(p), A^{\prime}(p)\right)^{2},
$$

where \#- denotes a set cardinal and $\widetilde{P}_{K}^{J} \subset P_{K}^{J}$ is the set obtained by discarding the worst pairings. The most time-consuming part of the proposed spatial registration process is the computation of the point pairing function $\phi_{A}$, which is proportional to the number of points to be paired (i.e. $\# P_{K}^{J}$ ) and to the cost of the nearest neighbor search (function of $\# P_{K}^{I}$ ). First, surfaces $S_{K}^{J}$ can be sub-sampled using feature parcellation [11] to decrease the computational time. Second, we privileged a k-d tree representation [12] of the set of points $P_{K}^{I}$ since the nearest neighbor research computational cost is logarithmic with this structure. Finally, the spatial registration computational cost is $O\left(\# P_{K}^{J} \ln \left(\# P_{K}^{I}\right)\right)$. Typically, if $P_{K}^{J}$ is a parceling of $S_{K}^{J}$ of about $10^{4}$ points and $P_{K}^{I}$ is represented as a k-d tree of about $5 \cdot 10^{5}$ points, the ICP process converges in about 20 seconds.

\section{TEMPORAL REGISTRATION}

We now consider a temporal sequence of 3D images $\left\{I_{t_{i}}\right\}_{i \in\{0: n\}}$ and a still 3D image $J$ imaged from two distinct FM. The aim of the temporal registration step is to extract the time-point $t_{J} \in\left[t_{0}, t_{n}\right]$ that achieves the best spatial registration of $J$ into the sequence. Although the acquisition techniques offer the means for imaging with a temporal resolution good enough to enable cell filiation extractions, the morphological deformations remain important between successive images. In order to ensure a precise temporal registration, a preliminary step is to refine the sequence temporal resolution (section 4.1$)$ by building a "continuous-like" movie $\left\{S_{K}^{I}(t)\right\}_{t \in\left[t_{0}, t_{n}\right]}$ from the original temporally sparse sequence $\left\{S_{K}^{I_{t_{i}}}\right\}_{i \in\{0: n\}}$. Then, we process to the so-called temporal registration step (section 4.2).

\subsection{Sequence temporal resolution enhancement}

The following three-fold process is repeated for $i \in\{0: n-1\}$ :

1. Estimate of the non-linear transformation $\mathbf{T}_{t_{i} \leftarrow t_{i+1}}$ that resamples $I_{t_{i}}$ on $I_{t_{i+1}}$;

2. Estimate of intermediary transformations $\left\{\mathbf{T}_{t_{i} \leftarrow t}\right\}_{t \in\left[t_{i}, t_{i+1}\right]}$;

3. Building surface movie $\left\{S_{K}^{I}(t)\right\}_{t \in\left[t_{i}, t_{i+1}\right]}$;

The complete movie $\left\{S_{K}^{I}(t)\right\}_{t \in\left[t_{0}, t_{n}\right]}$ is finally obtained by juxtaposing the reconstructed surface movies.

\subsubsection{Transformation $\mathbf{T}_{t_{i} \leftarrow t_{i+1}}$}

In order to deal with the plant rigid movements that may occur between two acquisitions, we first compute a rigid registration between $I_{t_{i}}$ and $I_{t_{i+1}}$. In the following, we assume that this rigid motion has been compensated for in the original sequence.

We then compute an affine transformation $\mathbf{A}_{t_{i} \leftarrow t_{i+1}}$ that linearly registers $I_{t_{i}}$ and $I_{t_{i+1}}$. This affine transformation is then used as an initialization for the non-linear transformation (encoded by a vector-field) $\mathbf{T}_{t_{i} \leftarrow t_{i+1}}=\mathrm{Id}+\mathbf{u}_{t_{i} \leftarrow t_{i+1}}$ :

$$
\forall M \in \mathcal{D}\left(I_{t_{i+1}}\right), \mathbf{T}_{t_{i} \leftarrow t_{i+1}}(M)=M+\mathbf{u}_{t_{i} \leftarrow t_{i+1}}(M),
$$

where $\mathcal{D}(I)$ is the domain of the image $I$. We computed the rigid, affine and non-linear transformations using the block matching framework [13], which can be described as an ICP-like iterative method where pairings are built by optimizing a similarity metric between blocks of the images to be registered. However, experience has shown that when the deformations are too important between successive images of the sequence (because of a low temporal resolution), the non-linear registration may fail. In this case, one can use an alternative method as [7] that estimates the meristem cell lineages while refining a non-linear deformation.

\subsubsection{Intermediary transformations}

To compute the vector-field $\mathbf{u}_{t \leftarrow t_{i+1}}$, different models could be used. Here, we simply assume the linearity of the transformation progression between time-points $t_{i}$ and $t_{i+1}$. Considering the interpolation coefficient $\alpha=\frac{t-t_{i}}{t_{i+1}-t_{i}} \in[0,1]$, the vector-field is given by:

$$
\forall t \in\left[t_{i}, t_{i+1}\right], \mathbf{u}_{t \leftarrow t_{i+1}}=(1-\alpha) \mathbf{u}_{t_{i} \leftarrow t_{i+1}},
$$

Then the transformation $\mathbf{T}_{t_{i+1} \leftarrow t}$ is computed as the inverse transform of $\mathbf{T}_{t \leftarrow t_{i+1}}=\mathrm{Id}+\mathbf{u}_{t \leftarrow t_{i+1}}$. One can notice that assuming linearity of the transformation progression between two consecutive time-points, the vector-fields $\mathbf{u}_{t_{i} \leftarrow t}$ and $\mathbf{u}_{t_{i+1} \leftarrow t}$ are proportional and verify the relation

$$
\mathbf{u}_{t_{i} \leftarrow t}=-\frac{\alpha}{1-\alpha} \mathbf{u}_{t_{i+1} \leftarrow t},
$$

leading to $\mathbf{T}_{t_{i} \leftarrow t}=\mathrm{Id}+\mathbf{u}_{t_{i} \leftarrow t}$. 


\subsubsection{Building surface movie}

We use the transformations $\left\{\mathbf{T}_{t_{i} \leftarrow t}\right\}_{i, t}$ given by (5) to build for each $k \in K$ a continuous-like movie of surfaces $\left\{S_{k}^{I}(t)\right\}_{t \in\left[t_{0}, t_{n}\right]}$ from the temporally sparse sequence $\left\{S_{k}^{I_{t_{i}}}\right\}_{i \in\{0: n\}}$. In this work, we fix

$$
\begin{array}{r}
S_{k}^{I}\left(t_{i}\right)=S_{k}^{I_{t_{i}}} \quad \forall i \in\{0: n\}, \\
\left.S_{k}^{I}(t)=S_{k}^{I_{t_{i}}} \circ \mathbf{T}_{t_{i} \leftarrow t} \quad \forall t \in\right] t_{i}, t_{i+1}[.
\end{array}
$$

From now, the remaining problems are to determine for each $t$ the spatial transformation that registers the set of surfaces $S_{K}^{J}$ on the set $S_{K}^{I}(t)$ and to extract the optimal time-point $t_{J} \in\left[t_{0}, t_{n}\right]$ such that $S_{K}^{I}\left(t_{J}\right)$ fits the best the transformed set of surfaces $S_{K}^{J}$.

\subsubsection{Side result: images interpolation}

With the knowledge of both transformations $\mathbf{T}_{t_{i} \leftarrow t}$ and $\mathbf{T}_{t_{i+1} \leftarrow t}$ given by section 4.1.2, an interpolated image can be computed from the re-sampled images $I_{t_{i}}(t)=I_{t_{i}} \circ \mathbf{T}_{t_{i} \leftarrow t}$ and $I_{t_{i+1}}(t)=I_{t_{i+1}} \circ$ $\mathbf{T}_{t_{i+1} \leftarrow t}$ with the relation

$$
I(t)=(1-\alpha) I_{t_{i}}(t)+\alpha \cdot I_{t_{i+1}}(t)
$$

where $\alpha \in[0,1]$ is defined in section 4.1.2. Fig. 2 shows an image interpolation result. In the following, $\{I(t)\}_{t \in\left[t_{0}, t_{n}\right]}$ denotes the movie of interpolated images from the sequence $\left\{I_{t_{i}}\right\}_{i \in\{0: n\}}$. Although this interpolated movie does not present an interest for the specific problem of image-to-sequence spatio-temporal registration, it provides a continuous-like geometric interpretation of the meristem growth throughout the FM developing stages (Fig. 3). We used $\{I(t)\}_{t \in\left[t_{0}, t_{n}\right]}$ to illustrate our registration results.

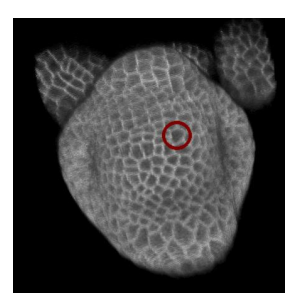

$I_{t_{i}}$

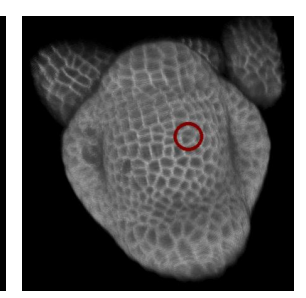

Interpolated $I(t)$

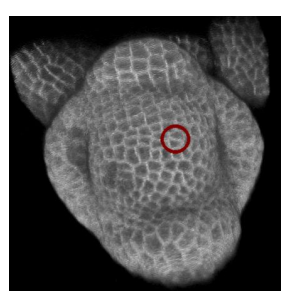

$I_{t_{i+1}}$
Fig. 2: Floral meristem interpolation result for $t=\frac{t_{i}+t_{i+1}}{2}$. Red circle highlights a cell division.

\subsection{Image-to-sequence temporal registration}

The temporal registration process consists of extracting the timepoint $t_{J}$ that optimizes a surface similarity criterion $s$ :

$$
t_{J}=\arg \max _{t \in\left[t_{0}, t_{n}\right]} s\left(S_{K}^{I}(t), A_{I(t) \leftarrow J}^{\mathrm{opt}}\left(S_{K}^{J}\right)\right) .
$$

The reader may notice that in order to compute the transformations $A_{I(t) \leftarrow J}^{\text {opt }}$, the proposed spatial registration method asks for a manual initialization of the transformation $A_{I(t) \leftarrow J}^{\text {init }}$. However, considering that the rigid movements of the movie are corrected (section 4.1.1), one can use the same transformation initialization for any time $t \in\left[t_{0}, t_{n}\right]$ of the movie, i.e. $A_{I(t) \leftarrow J}^{\text {init }}=A_{I \leftarrow J}^{\text {init }}$.

We propose to use a criterion built from the cumulative histogram of residual distances between paired points at the optimal transformation. Let us define the residual distance function res be- $_{t}$ tween two paired points after registration and the cumulative histogram of residual distances function $h_{t}$ :

$$
\begin{gathered}
\operatorname{res}_{t}: p \in P_{K}^{J} \mapsto \operatorname{dist}\left(\phi_{A_{I(t) \leftarrow J}^{\text {opt }}}(p), A_{I(t) \leftarrow J}^{\text {opt }}(p)\right) \in \mathbb{R}_{+}, \\
h_{t}: d \in \mathbb{R}_{+} \mapsto \#\left\{p \in P_{K}^{J}: \operatorname{res}_{t}(p) \leq d\right\} .
\end{gathered}
$$

The better the registration, the smaller the residuals. Thus, for a fixed distance value $d$, we expect that $h_{t}(d)$ will be maximal for $t=t_{J}$, meaning that $t_{J}$ is the time-point for which the transformation of the point-set $P_{K}^{J}$ best fits the target point-set $P_{K}^{I}\left(t_{J}\right)$. Let us denote $t_{J}(d)=\arg \max _{t} h_{t}(d)$. We define the optimal registration timepoint $t_{J}$ as the time-point that maximizes the most frequently the cumulative histogram $h_{t}(d)$ for values of $d$ taken in an adequate interval $D$ (Fig. 4), i.e. the most frequent value $t_{J}(d)$ over $d \in D$. In Figs 4 and 5 , we chose to plot a normalized surface $n(t, d)$ of the cumulative histograms $h_{t}(d)$ where $\forall d, t, n(t, d)=h_{t}(d) / h_{t_{J}(d)}(d)$ for a better visualization (hence $n(t, d) \in[0,1]$ and $n\left(t_{J}(d), d\right)=$ $1)$.

\section{EXPERIMENTS}

\subsection{Data}

To generate the data for imaging, we took wild type plants of Arabidopsis thaliana and introduced a modified yellow fluorescent protein that contained an acyl signal sequence at its $\mathrm{N}$-terminus. This acylated YFP clearly marked the edges of the cell for subsequent segmentation. Plants were initially grown in soil under continuous light until they made the transition from the vegetative to reproductive phase of growth. For microscopy, plantlets exhibiting an inflorescence stem of maximum length $1 \mathrm{~cm}$ were transferred in to a plastic box containing molten, cooled $1 \% \mathrm{w} / \mathrm{v}$ agar comprising 2.2 grams litre ${ }^{-1}$ MS salts and Gamborg B5 vitamins. Care was taken to also transfer as much of the root as possible. After meristem dissection to remove the obstructing flowers, plants were imaged using an upright Zeiss 780 or LSM700 confocal microscope equipped with a 20x water dipping objective lens. Confocal z-stacks were acquired of a region that encompassed a newly formed organ primordium with voxel dimensions set to no more than $300 \mathrm{~nm}^{3}$. Plantlets were kept in the light between time points.

A $3 \mathrm{D}+t$ sequence consists in 6 to $183 \mathrm{D}$ images. 3D stacks are made of 130 to 683 2D slices, with a slice thickness that may vary from 130 to $610 \mathrm{~nm}$. A 2D slice is typically of $400 \times 400$ pixels with a pixel size of about $250 \mathrm{~nm}$.

For the feature extraction step (section 2, Fig. 1), cell segmentations of a complete $3 \mathrm{D}+t$ sequence ( 7 timepoints) and of a still image from another sequence have been produced by MARS [7] and further checked by an expert and manually corrected if required.

\subsection{Inter-individual registration}

We worked on datasets from two FM for the inter-individual registration method demonstration. Given the surfaces $S_{K}^{J}$ from a still image (FM at developing stage 3 ) and the surface movies $\left\{S_{K}^{I}(t)\right\}_{t}$ (sampled every 2 hours) built from a sequence of 3D images (FM imaged from stage 2 to 4 during 55 hours), we detail here the spatio-temporal registration result of $S_{K}^{J}$ onto $\left\{S_{K}^{I}(t)\right\}_{t}$. We fixed the distance set $D$ to 30 uniformly sampled values between $0 \mu \mathrm{m}$ and $12 \mu \mathrm{m}$. Fig. 5 presents the registration result that was assessed by visual inspection and considered as correct by biologists. We compared the robustness 


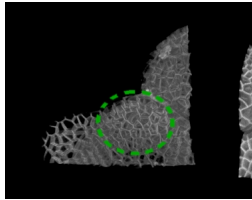

Stage 1

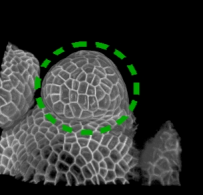

Stage 2

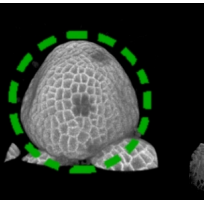

Stage 3

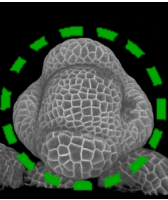

Stage 4

Fig. 3: Images from a sequence of Arabidopsis thaliana FM morphogenesis (inside the green circle) at development stages ranging from stage 1 (no clear distinction between the FM and the SAM) to stage 4 (sepals overlie FM).

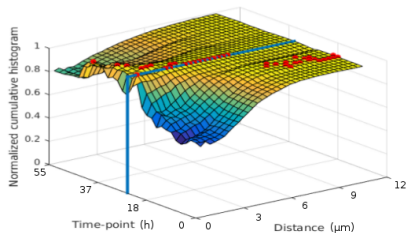

(a)

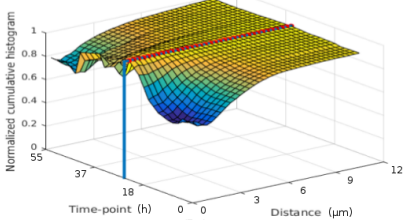

(b)

Fig. 4: Inter-individual temporal registration. Normalized cumulative histogram $(t, d) \mapsto n_{t}(d)$ for: (a) $K=\{1\}$ (only interface Background-L1) and untrimmed LSE optimization; (b) $K=\{1,2\}$ (interfaces BackgroundL1 and L1-L2) and trimmed LSE optimization. The red dots correspond to points $\left(t_{J}(d), d\right)$ where $n\left(t_{J}(d), d\right)=1$.

of the temporal registration result for different spatial registration parameters (untrimmed (U-) and trimmed (T-) LSE with $K=\{1\}$, then trimmed LSE with $K=\{1,2\}$ ). The results, synthesized bellow and illustrated by Fig. 4, demonstrate the importance to consider both a robust ICP instead of a basic one and several layer interfaces instead of only one for the spatial registration:

- U-LSE, $K=\{1\}: t_{J}(d)=t_{J} \pm 2 \mathrm{~h}$ for $50 \%$ of $d \in D$ values;

- T-LSE, $K=\{1\}: t_{J}(d)=t_{J} \pm 2 \mathrm{~h}$ for $83 \%$ of $d \in D$ values;

- T-LSE, $K=\{1,2\}: t_{J}(d)=t_{J}$ exactly for $100 \%$ of $d \in D$ values.

\section{CONCLUSION}

We presented a method for spatio-temporal registration of plant developing flowers at cell shape resolution. We showed the ability of the proposed method to spatially and temporally register one still

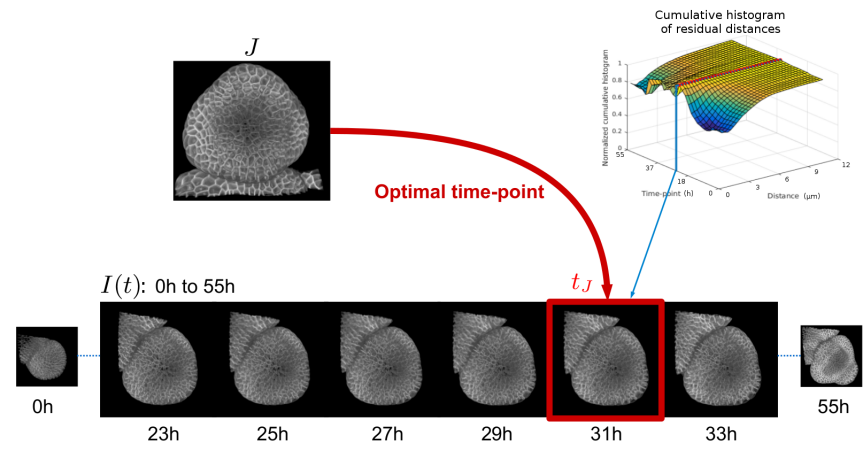

Fig. 5: Inter-individual temporal registration result with 3D views of the registered meristem and the interpolated movie at several time-points.

image onto a $3 \mathrm{D}+t$ sequence of images by considering invariant features, namely the cell epidermal and sub-epidermal layers L1 and L2. Such an image-to-sequence registration tool offers the means to build a 4D template (spatio-temporal dimensions) on which every individual can be projected, opening the avenue to the statistical analysis of population.

Future investigations will consist in performing more experiments to first assess the validity of the proposed method and second to build a $3 \mathrm{D}+t$ template from several individuals. Special attention should be paid to a dedicated biological validation of the registration results. Ongoing technical work is conducted on the surface movie reconstruction that will be more elegant with a true surface interpolation between two time points. Using more complex registration techniques [7] will also enables to deal with more sparsely sampled temporal sequences.

Acknowledgment: Authors are funded by the following grants: IPL Morphogenetics (GM), ERC Morphodynamics (JT and YR), ANR Dig-Em (GM and CG), ANR IBC (CG), HFSP project BioSensors \#RGP0054/2013 (CG).

\section{REFERENCES}

[1] PJ Keller, "Imaging Morphogenesis: Technological Advances and Biological Insights," Science, vol. 340, no. 6137, pp. 1234168+, June 2013

[2] L Guignard, C Godin, UM Fiuza, L Hufnagel, P Lemaire, and G Malandain, "Spatio-temporal registration of embryo images," in ISBI, Beijing, China, Apr. 2014, IEEE.

[3] G Michelin, L Guignard, UM Fiuza, P Lemaire, C Godin, and G Malandain, "Cell Pairings for Ascidian Embryo Registration," in ISBI, New York, United States, Apr. 2015, IEEE.

[4] MK Barton and RS Poethig, "Formation of the shoot apical meristem in arabidopsis thaliana: an analysis of development in the wild type and in the shoot meristemless mutant," Development, vol. 119, no. 3, pp. 823-831, 1993.

[5] J Legrand, Toward a multi-scale understanding of flower developmentfrom auxin networks to dynamic cellular patterns, Ph.D. thesis, Lyon, École normale supérieure, 2014.

[6] B Landrein, Y Refahi, F Besnard, N Hervieux, V Mirabet, A Boudaoud, T Vernoux, and O Hamant, "Meristem size contributes to the robustness of phyllotaxis in arabidopsis," Journal of experimental botany, p. eru482, 2014.

[7] R Fernandez, P Das, V Mirabet, E Moscardi, J Traas, JL Verdeil, G Malandain, and C Godin, "Imaging plant growth in 4-D: robust tissue reconstruction and lineaging at cell resolution," Nat Meth, vol. 7, pp. 547-553, 2010.

[8] G Michelin, L Guignard, U Fiuza, and G Malandain, "Embryo Cell Membranes Reconstruction by Tensor Voting," in ISBI, Beijing, China, Apr. 2014, IEEE.

[9] PJ Besl and ND McKay, "A method for registration of 3-D shapes," IEEE T Pattern Anal, vol. 14, no. 2, pp. 239-256, 1992.

[10] PJ Rousseeuw and AM Leroy, Robust Regression and Outlier Detection, John Wiley \& Sons, New York, NY, USA, 1987.

[11] G Flandin, F Kherif, X Pennec, D Rivière, N Ayache, and JB Poline, "Parcellation of brain images with anatomical and functional constraints for fmri data analysis," in ISBI, Washington, United States, 2002, pp. 907-910, IEEE.

[12] JL Bentley, "Multidimensional binary search trees used for associative searching," Comm. of the ACM, vol. 18, no. 9, pp. 509-517, 1975.

[13] S Ourselin, A Roche, S Prima, and N Ayache, "Block matching: A general framework to improve robustness of rigid registration of medical images," in MICCAI. 2000, vol. 1935 of LNCS, pp. 557-566, Springer. 